\title{
The effects of irrelevant information and stimulus dominance in an oddity problem '
}

\author{
DANIEL S. LORDAHL, STEPHEN E. BERGER AND ALEXAHDER A. MANNING
}

UNIVERSITY OF MIAMI

College students solved multidimensional oddity problems with 3, 4 or 5 stimulus dimensions, only one of which was relevant. In one treatment, the differences between the two levels of each stimulus dimension were set to be about equal, perceptually. In the second treatment, one of the dimensions had a more apparent difference between levels than for the other dimensions. The results indicated that Ss tested an increasing number of oddity hypotheses as the number of stimulus dimensions increased, and that trials to criterion were less when the relevant dimension was perceptually dominant than when it was perceptually equal to other dimensions.

In previous work using the multidimensional oddity problem (Lordahl, 1965, 1967), the influence of highly dominant stimulus dimensions was found to be greater than the effects due to the number of dimensions varied in the task. Therefore, the stimuli used in the present study were scaled in pilot work so that stimulus dimensions could be used which had known degrees of perceptual dominance. It was hoped that such control over stimulus features would permit the expected effects of irrelevant information to be clearly demonstrated in this task as they have been in concept identification problems (Archer, Bourne, \& Brown, 1955; Lordahl, 1961).

\section{Stimuli and rask}

Details of the apparatus and task are given more fully in Lordahl (1967). The stimuli for a five dimension problem are illustrated in Fig. 1; note the bileveled dimensions of circle size, orientation of the circles, flag angle, circle filling, and dot size. Problems with three dimensions varied the first three listed, and those with four dimensions included the first four listed. As in Fig. 1, one of the three patterns presented to a $S$ on each trial was always odd with respect to any dimension considered.

The task was organized into blocks of five trials, on each trial the $S$ being required to select one of the patterns by means of pulling a corresponding lever. No feedback was given until all three trials of each block were completed. Then the S was told "correct" and a green light lighted if and only if all three responses had been correct. If one or more errors had been made in the block, the word "wrong"' was spoken by $\mathrm{E}$.

The oddity solution always required that a single dimension be used, the correct pattern being the odd pattern present with respect to the relevant dimension. Ss were informed of the nature of the feedback con- tingencies, the fact of a single relevant dimension, and were given a simple example of the oddity concept. All 144 Ss whose data are used were run to a criterion of four consecutive errorless blocks. Another six Ss failed to solve the problem.

Stimuli were randomly arranged with the restrictions that some pattern on each trial contain an odd level of each dimension and that no two dimensions would yield the same pattern of responses, when used with the oddity principle, within any block of three trials. Design

In an orthogonal design, the number of stimulus dimensions, three, four, or five, was combined with two types of stimuli, balanced dimensions and unbalanced dimensions. The unbalanced condition contained stimuli which had flag angles of 0 and 45 degrees, a much more striking perceptual difference than for the other dimensions. The balanced stimuli contained dimensions of approximately equal perceptual dominance. Orthogonal to these factors, three different problems were used, specifically, either circle size, orientation, or flag angle was relevant. Within each of the resultant 18 treatment conditions eight Ss were run, five by one $\mathrm{E}$ and three by another. All Ss were assigned to conditions at random, within the above constraints.

\section{Resulis and Discussion}

Ss were each scored on the basis of two dependent measures, trial blocks to reach the criterion run, and the number of response sequences (over a block of three trials) which matched the oddity pattern of some dimension on that block. The latter measure will be termed oddity responses. The first block of the criterion run was included in the counting of oddity responses.

Since the 27 possible response sequences in a block coincided with three, four, or five oddity patterns in the three, four, and five dimension problems, the number of oddity responses for each $S$ was corrected for chance, using the standard notion of an observed

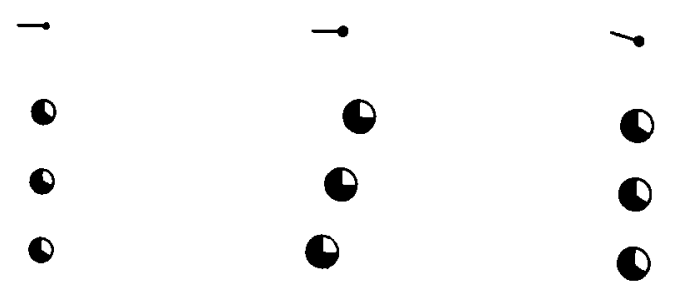

Fig. 1. An example of a stimulus from a balanced, five dimension problem. 
score being composed of a true score plus a number of correct guesses.

Both dependent variables produced gross heterogeneity of variance within the cells of the design. In each case, a square root transformation was used to correct this statistical problem. Means given here are based on the transformed measures, as are the statistical tests.

In terms of the trials to criterion measure, the number of dimensions in the stimuli did not significantly affect performance. The means for three, four, and five dimension conditions were 2.47, 2.51, and 2.75, respectively, the $F$ for the linear trend being 1.11, df $=1 / 108$. However, with the same measure, the interaction of relevant dimensions and balance was significant at the .05 level $(F=4.02, d f=2 / 108)$. The difference is caused specifically by the unbalanced dimension (flag angle) which had a mean of 2.07 when relevant and perceptually dominant, compared to 2.94 when relevant and of equal dominance. The mean for the other conditions combined was 2.61 .

In terms of the transformed number of oddity responses, the number of dimensions used significantly affected performance. The means were $2.00,2.20$, and 2.40, for the three, four, and five dimension conditions, respectively. The corresponding $F$ for the linear trend of these means was $5.15(\mathrm{df}=1 / 108, p<.025)$.

The balance by relevant dimension interaction was not significant in this analysis.

In both of the above analyses, a significant interaction of $\mathrm{E}$ by balance by number of dimensions was found. This interaction effect appears anomalous and is tentatively interpreted as a sampling error.

No other sources of variation, including $E$ effects, yielded significance at the .05 level in either analysis.

Apparently, the effect of number of stimulus dimensions is not a simple one. When testing oddity hypotheses, the Ss show the expected increase in number of hypotheses tested as the number of dimensions is increased from three to five. However, the total number of trials to solution is not influenced to a significant degree. The reverse is the case for the perceptual dominance of the flag dimension. When considering trials to criterion, the variation in perceptual dominance led to significant changes in performance. The same conditions did not produce significant changes in number of oddity hypotheses tested.

Two additional sources of information provide the basis for a suggested interpretation of these findings: The number of Ss who reported using combinations of dimensions for the formulation of hypotheses was greatest in the three dimension conditions, and the pooled within cells variance of trials to criterion (untransformed) was greatest in the three dimension conditions. It is suggested that Ss in the three dimension conditions tended to Ignore the instructions that combinations of dimensions were not important and to generate such complex hypotheses. In contrast, the five dimension materials may have been perceptually complex enough to deter Ss from such attempts. An appropriate method of scoring response sequences to measure types of complex hypotheses is being developed and may allow more direct verification of this suggested tendency.

\section{References}

Archer, E. J., Boume, L. E., Jr., \& Brown, F. G. Concept identification as a function of irrelevant information and instructions. J. exp. Psychol., 1955, 49, 153-164.

Lordahl, D. S. Concept identification using simultaneous auditory and visual signals. $J$. exp. Psychol., 1961, 62, 283-290.

Lordahl, D. S. Hypothesis behavior in a multidimensional oddity problem. Psychon. Sci., 1965, 3, 229-230.

Lordahl, D. S. The effect of irrelevant information in a multidimensional oddity task. Psychon. Sci., 1967, 7, 141-142.

\section{Nore}

1. This work was supported by grant No. MH 10277 from the National Institute of Mental Health, U. S. Public Health Service. 